\title{
Performance of Galileo-only dual-frequency absolute positioning using the fully serviceable Galileo constellation
}

\author{
Tomasz Hadas $^{1}$ (1) $\cdot$ Kamil Kazmierski $^{1} \cdot$ Krzysztof Sośnica $^{1}$
}

Received: 8 March 2019 / Accepted: 2 August 2019 / Published online: 7 August 2019

(c) The Author(s) 2019

\begin{abstract}
The recent development of the Galileo space segment and the accompanying support of the International GNSS Service (IGS) allows for worldwide Galileo-only positioning. In this study, different techniques of dual-frequency absolute positioning using the fully serviceable Galileo constellation are evaluated for the first time and compared to the performance of GPS positioning. The daily static positioning based on the broadcast ephemeris using Galileo pseudoranges is significantly more accurate than the corresponding GPS solutions, obtaining the accuracy of a few decimeters. In the kinematic mode, the accuracy is better than $10 \mathrm{~m}$ and $20 \mathrm{~m}$ for the horizontal and vertical components, respectively, which is comparable to that of GPS. Precise absolute positioning using pseudorange and carrier phase Galileo observations combined with IGS Real-Time Service (RTS) or Multi-GNSS Experiment products is not yet as good as the corresponding GPS solutions. In the static mode, the root mean squared error (RMSE) between estimated and reference coordinates does not exceed $0.05 \mathrm{~m}$ and $0.06 \mathrm{~m}$ for the horizontal and vertical components, respectively. In the kinematic mode, the respective accuracies are better than $0.17 \mathrm{~m}$ and $0.21 \mathrm{~m}$. Moreover, we show that both GPS and Galileo pseudorange solutions benefit from the RTS when compared to the broadcast solutions with the improvement in the accuracy between 10 and 59\%. Remarkable results are achieved for Galileo Precise Point Positioning (PPP) solutions based on the broadcast ephemeris. In the static mode, the RMSE is 0.07 and $0.10 \mathrm{~m}$ for the horizontal and vertical components which is three and two times better, respectively, then the corresponding solutions based on GPS.
\end{abstract}

Keywords Galileo $\cdot$ Real-time $\cdot$ Positioning $\cdot$ RTS $\cdot$ MGEX

\section{Introduction}

The original idea of the GNSS was to determine an instantaneous position for navigation based on pseudorange observations and broadcast ephemeris delivered by at least 4 satellites (Parkinson and Axelrad 1988). This basic method, called Standard Point Positioning (SPP), allows for obtaining coordinates using one epoch of observations with the accuracy of several meters (Satirapod et al. 2001; Cai et al. 2014a). Single-frequency instantaneous SPP solution neglecting ionosphere delay can reach $4.5 \mathrm{~m}$ and $7 \mathrm{~m}$ accuracy for GPS-only and BeiDou-only, respectively (Odolinski et al. 2014). Single-epoch Galileo SPP solutions neglecting

Tomasz Hadas

tomasz.hadas@upwr.edu.pl

1 Institute of Geodesy and Geoinformatics, Wroclaw University of Environmental and Life Sciences,

Grunwaldzka 53, 50-357 Wrocław, Poland ionosphere delay achieve $6.6 \mathrm{~m}$ positioning accuracy in 3D and about $3 \mathrm{~m}$ accuracy when the influence of ionosphere is eliminated by models (Angrisano et al. 2013). The very initial SPP tests for four IOV Galileo and GPS satellites achieve an accuracy of about $2.5 \mathrm{~m}$, which corresponded to a $10 \%$ improvement when compared to GPS-only SPP solution (Cai et al. 2014b). The use of precise orbit and clock products from the International GNSS Service (IGS) and averaging static dual-frequency SPP solutions over $24 \mathrm{~h}$ a gives accuracy at the 1-m level for all components (Satirapod et al. 2001). Nevertheless, the accuracy of both, the instantaneous and the static SPP, is insufficient for many applications.

Real-Time Kinematic (RTK) is a method attempting to satisfy high precision positioning. A reference station or a reference network provides real-time corrections which, however, is expensive to establish and maintain. The accuracy of GPS-only, Galileo-only and GPS + Galileo RTK 
solution baselines up to $99 \mathrm{~km}$ is better than $1 \mathrm{~cm}$ (Paziewski and Wielgosz 2014).

Trying to overcome network dependence, the concept of Precise Point Positioning (PPP) was introduced (Malys and Jensen 1990; Zumberge et al. 1997). Several centimeters accuracy results in the post-processing mode of this technique were proven by many researchers (Cai et al. 2015; Fu et al. 2019). Recently developed algorithms for ambiguity fixing allowed increasing the positioning accuracy to the level of several millimeters (Liu et al. 2019).

The precise multi-GNSS positioning requires products that support newly established systems. Thanks to the MultiGNSS Experiment (MGEX, Montenbruck et al. 2017), the GNSS community can explore multi-constellation solutions. However, the latency of MGEX products does not meet the requirements of real-time precise GNSS applications. Therefore, the IGS established the Real-Time Service (RTS), which provides combined corrections for GPS and GLONASS. Moreover, Centre National d'Études Spatiales (CNES) Analysis Center supports all available GNSS. Therefore, using a receiver capable of tracking carrier phase measurements multi-GNSS PPP can be obtained in real-time mode, i.e., using broadcast ephemeris with or without RTS corrections, as well as in post-processing mode, e.g., using final products.

Although there is much research describing different approaches of multi-GNSS positioning, there has been little research and no up-to-date results for Galileo-only positioning, primarily due to insufficient number of usable Galileo satellites. The current large number of usable Galileo satellites and the availability of supporting products finally allow us to perform Galileo-only positioning using SPP and PPP techniques, both in real-time and post-processing mode.

The main goal of this study is to assess the performance of pseudorange and carrier phase dual-frequency static and kinematic positioning, thus fulfilling the existing gap in the evaluation of Galileo-only absolute positioning. The accuracy of the Galileo broadcast ephemeris is about $20 \mathrm{~cm}$, which is three times better than that of GPS (Montenbruck et al. 2018). This creates new potential opportunities of PPP solutions that do not require any further corrections. Hence, we present results of pseudorange positioning supported by RTS corrections and PPP solutions based on the broadcast ephemeris instead of precise orbit and clock products. We compare Galileo-only solutions with GPS-only solutions.

\section{Galileo status}

The Galileo constellation consists of Medium-Earth Orbiters (MEO) distributed among 3 orbital planes. Each orbital plane named A, B and C nominally should be occupied by 8 operational pieces of spacecraft in circular orbits (Píriz

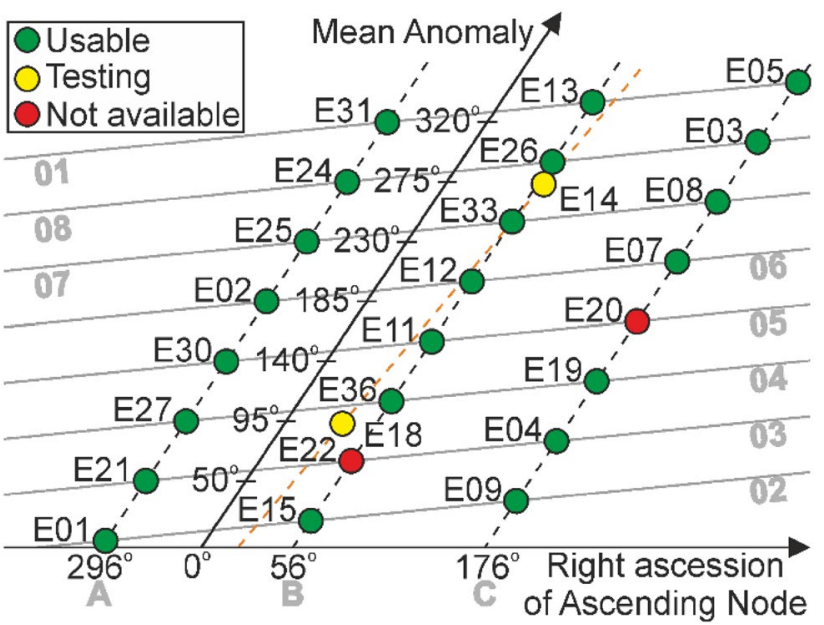

Fig. 1 Galileo constellation status as of January 1, 2019. Satellites are marked with PRN numbers. Gray letters and numbers identify a satellite slot

et al. 2005). The first spacecraft of the current Galileo constellation was launched at the end of 2011. After a very slow orbit populating, the last 2 years brought significant progress. During each of three launches, four new satellites were simultaneously moved to the orbit by Ariane 5 (Chatre and Verhoef 2018). The initial expectation was that all the Galileo satellites would take their final orbital position by the end of October 2018 and enter into service in early 2019. On February 11, 2019, the newest four satellites E11, E13, E15, and E33 were announced healthy and are currently in the operational use (URL: https://www.gsa.europa.eu/ newsroom/news/latest-batch-galileo-satellites-enters-servi ce). Although during the test period these 4 satellites were still under commissioning status, they were broadcasting ephemeris data.

The first pair of the FOC satellites (E14, E18) missed their target and are in elliptical orbits (Delva et al. 2015). The E14 and E18 have abnormal eccentricity, the deviation of the semimajor axis from the nominal value equal to 0.16 and $1620 \mathrm{~km}$, respectively. Therefore, almanacs for E14 and E18 are not broadcast, because these two orbital parameters do not fit in the range of values foreseen in the Galileo Interface Control Document (Steigenberger and Montenbruck 2017).

Additionally, two other satellites, E20 and E22, are unserviceable. The first one transmits a single-frequency signal (Steigenberger and Montenbruck 2017) and the latter has been removed from the active service for constellation management purposes. Therefore, the present Galileo constellation consists of 22 properly functioning satellites, 2 testing satellites on improper orbits, and 2 unserviceable satellites (Fig. 1). Further launches are needed in order to place 

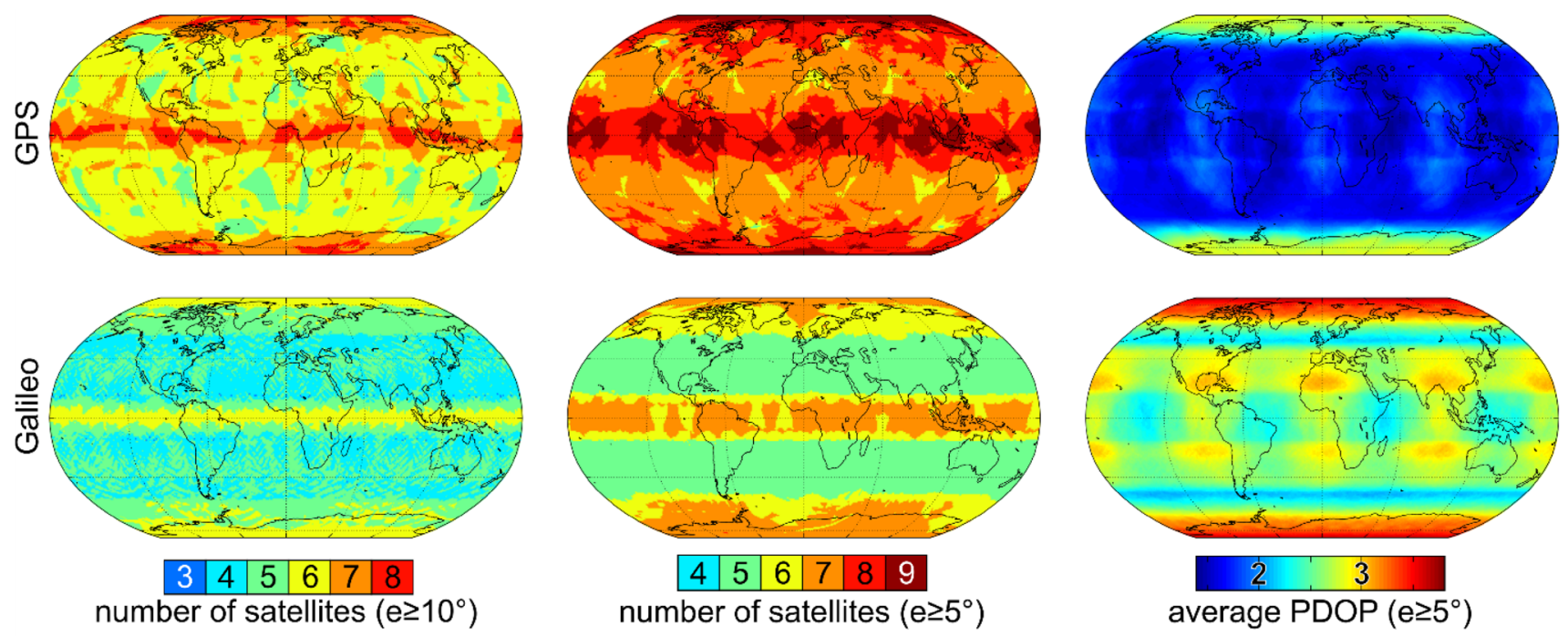

Fig. 2 Minimum number of visible satellites and PDOP for GPS (top) and Galileo (bottom). Number of satellites with an elevation mask $10^{\circ}$ (left) and $5^{\circ}$ (central). Average PDOP for elevation mask of $5^{\circ}$ (right). The data span is January 1-7, 2019

back-up satellites in orbit (URL: https://www.gsa.europa.eu/ galileo-initial-services).

In order to use satellites in positioning applications, it is necessary to receive their broadcast ephemeris. However, it is necessary to use orbits and clocks with corrections that are provided to the user with minimum latency to increase the real-time positioning quality. Such corrections are available in the predicted part of the IGS ultra-rapid products (Elsobeiey and Al-Harbi 2016) or in real-time corrections, which are provided in CLK93 stream (Loyer et al. 2012). Although broadcast ephemerides are available for 24 Galileo satellites, the real-time corrections are available only for 18 . This is because there are still no real-time orbit and clock corrections for the newest four satellites.

\section{Satellite availability and PDOP}

We used Galileo broadcast ephemeris and calculated Galileo satellite positions every 5 min from January 1 to 7, 2019, according to the Galileo Interface Control Document (European Space Agency 2010). Corresponding calculations were done for GPS. The surface of the earth was subdivided with a $1^{\circ} \times 1^{\circ}$ grid with zero altitudes, and for the cell midpoints we computed the elevations of the satellites. Then, for several elevation masks and assuming no obstructions in view, we calculated the number of visible satellites each epoch and the corresponding position dilution of precision (PDOP) parameter. This allowed us to determine the minimum number of satellites and the average PDOP for each grid cell globally. For global averaging, we used an area-weighted mean, in order to account for a different area of a grid cell.
We applied a PDOP threshold of 20 and skipped epochs when fewer than four satellites were in view, assuming the constellation geometry meeting these conditions was insufficient for reliable positioning. Therefore, we also provide the percentage of epochs with insufficient geometry, with at least four and five satellites in view. The percentage of epochs with PDOP $\leq 6$ is also given, as this threshold value is used in GPS PDOP analysis (Kaplan and Hegarty 2017).

During the test week, at least four Galileo satellites were visible above $10^{\circ}$ elevation mask almost everywhere and anytime. Fewer satellites were visible only at some individual epochs and spots, mainly along the $15^{\circ} \mathrm{N}$ and $15^{\circ} \mathrm{S}$ latitudes (Fig. 2, bottom left). At least six satellites were permanently in view above the equator and around the poles. If the elevation mask was lowered to $5^{\circ}$, then at least five satellites were in view anywhere at anytime. For some regions around the equator and the poles, at least seven satellites were permanently in view (Fig. 2, bottom central). For GPS, usually two more satellites were in view than for Galileo, reaching up to 9 satellites around the equator and the poles for the elevation mask of $5^{\circ}$ (Fig. 2, top left and top central). The global variability of average PDOP has also a longitudinal character. For the elevation mask of $5^{\circ}$, the average PDOP for GPS was usually lower than 2.0, except pole regions with average PDOP reaching 2.8 (Fig. 2, top right). For Galileo, the average PDOP varies from 2.1 to 3.0, except for polar regions with average PDOP reaching 3.8 (Fig. 2, bottom right).

Figure 3 presents satellite visibility for different elevation angles. It is worth noting that the maximum number of 14 visible Galileo satellites was found at some spots near Antarctica. A minimum of three and five satellites 


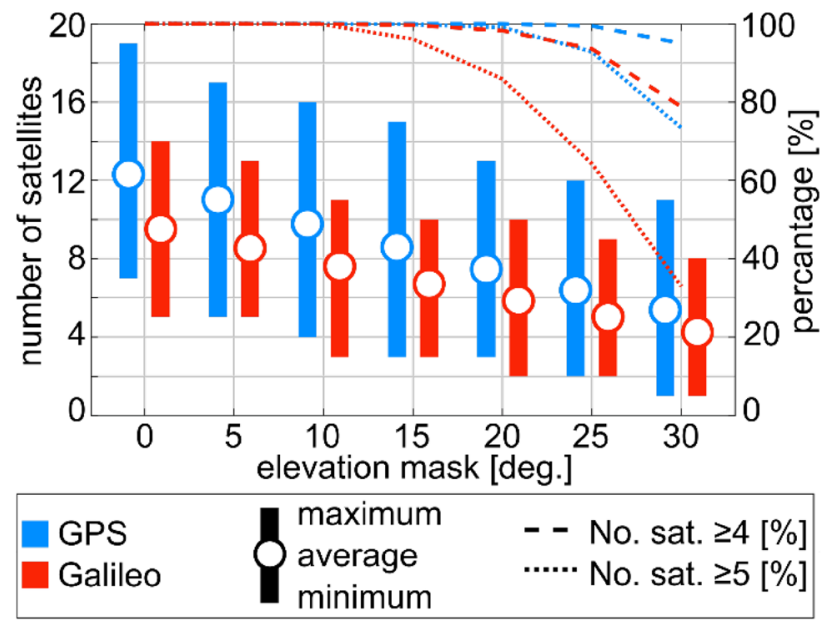

Fig. 3 Number of GPS and Galileo satellites in view and percentage of epochs with at least 4 and 5 satellites in view. The data span is January 1-7, 2019

was always visible when the elevation mask was not higher than $10^{\circ}$ and $5^{\circ}$, respectively. The average number of visible Galileo satellites for the elevation mask of $0^{\circ}$ was 9.5 and decreased to 4.3 for the elevation mask of $30^{\circ}$. For GPS the average number of visible satellites was larger by 1.1 and 2.8 , for elevation mask of $30^{\circ}$ and $0^{\circ}$, respectively. At least four and five Galileo satellites were visible in more than $98 \%$ of cases for elevation mask of $20^{\circ}$ and $10^{\circ}$, respectively. For GPS at least four and five satellites were visible in more than $99 \%$ of cases for the elevation mask of 25 and 20, respectively. Therefore, GPS positioning in urban-canyons should still outperform Galileo positioning.

For GPS, the global PDOP of 6 or less for the elevation mask of $5^{\circ}$ was available at least $99.8 \%$ of the time, which is above the GPS target performance metrics (https ://www.gps.gov/systems/gps/performance/). For Galileo, this metric was equal to $97.2 \%$. Therefore, when compared to GPS, the performance of Galileo is still slightly worse. However, in 2017 the average Galileo-only PDOP with $10^{\circ}$ elevation mask ranged from 6.0 to 8.2 (Pan et al. 2017), so the current average PDOP of 3.9 is almost twice as good. Figure 4 shows that average PDOP for Galileo was slightly larger than that for GPS for elevation masks up to $25^{\circ}$. Although we noticed a contrary effect for higher elevation masks, we attribute this to the increased number of epochs with insufficient Galileo geometry, i.e., $66 \%$ of epochs with fewer than four satellites in view and $60 \%$ of epochs with PDOP $>20$. PDOP $>20$ never occurred for Galileo and GPS when elevation mask was equal $5^{\circ}$ and $10^{\circ}$, respectively.

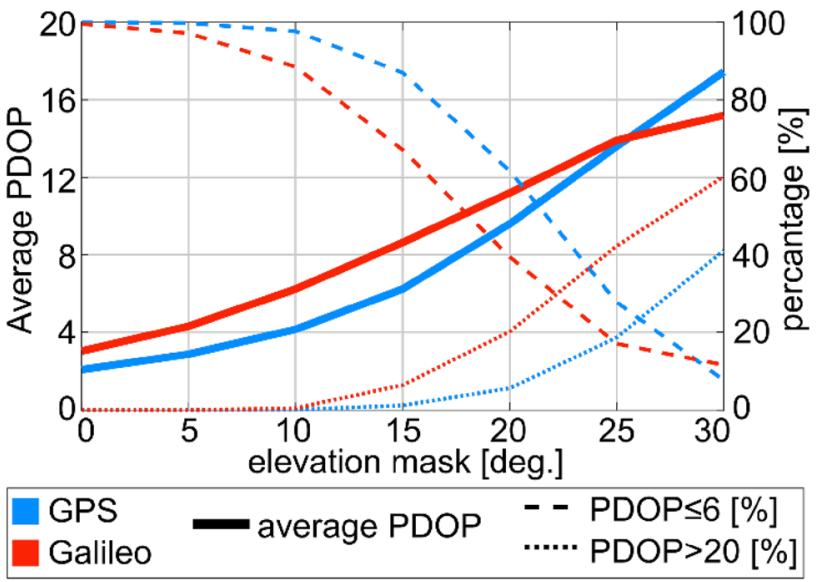

Fig. 4 Global average PDOP for GPS and Galileo and percentage of epochs with PDOP up to 6 and with PDOP above 20. The data span is January 1-7, 2019

\section{Methodology of Galileo-only positioning}

In the following subsections, the dual-frequency functional model for Galileo-only positioning is introduced. Details on the processing strategy are provided, and the selection of positioning variants is justified.

\section{Functional model}

We process dual-frequency data using the undifferenced and uncombined functional mode (Schönemann 2014), which includes multi-frequency pseudorange (code) $C$ and carrier phase $L$ observations, without forming any linear combinations:

$C_{i}^{s}-\rho_{0}^{s}+c \delta t^{s}+b_{C, i}^{s}=\boldsymbol{e}_{r}^{s} \cdot \delta \boldsymbol{X}_{\boldsymbol{r}}+c \delta t_{r}+m^{s} T_{Z}+\mu_{i} I^{s}$

$L_{i}^{s}-\rho_{0}^{s}+c \delta t^{s}+b_{L, i}^{s}=\boldsymbol{e}_{\boldsymbol{r}}^{s} \cdot \delta \boldsymbol{X}_{\boldsymbol{r}}+c \delta t_{r}+m^{s} T_{Z}-\mu_{i} I^{s}+\lambda_{i} N_{i}^{s}$

with

$\boldsymbol{e}_{\boldsymbol{r}}^{\boldsymbol{s}}=\left[\begin{array}{lll}\frac{X_{r}-X^{s}}{\rho_{0}^{s}} & \frac{Y_{r}-Y^{s}}{\rho_{0}^{s}} & \frac{Z_{r}-Z^{s}}{\rho_{0}^{s}}\end{array}\right]$

$\boldsymbol{\delta} \boldsymbol{X}_{\boldsymbol{r}}=\left[\begin{array}{lll}\delta X_{r} & \delta Y_{r} & \delta Z_{r}\end{array}\right]^{\mathrm{T}}$

$\mu_{i}=f_{1}^{2} / f_{i}^{2}$

$\rho_{0}^{s}=\sqrt{\left(X^{s}-X_{r, 0}\right)^{2}+\left(Y^{s}-Y_{r, 0}\right)^{2}+\left(Z^{s}-Z_{r, 0}\right)^{2}}$

where $i$ is the number of the frequency $f ; s$ identifies a satellite; $c$ is the speed of the light in vacuum; $\rho_{0}$ is the geometric 


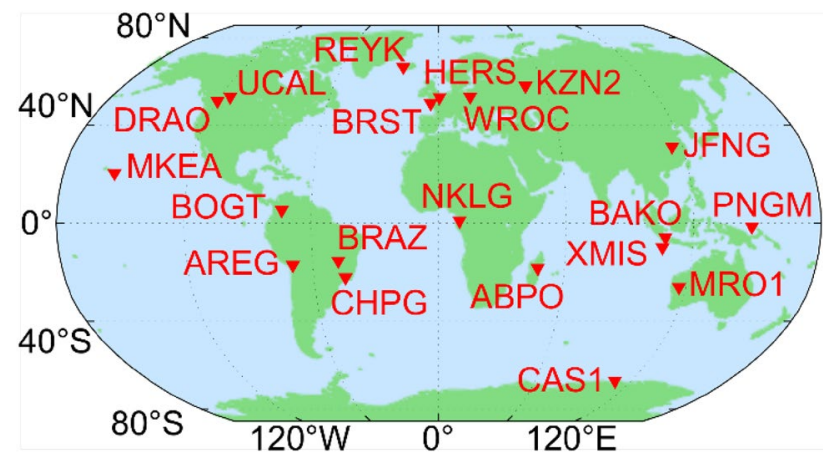

Fig. 5 Location of test stations

distance between the position of the satellite antenna phase center $\left(X^{s}, Y^{s}, Z^{s}\right)$ and the a priori position of the user antenna phase center $\left(X_{r}, Y_{r}, Z_{r}\right) ; \delta t_{r}$ and $\delta t^{s}$ are receiver and satellite clock offsets, respectively; $b_{C}$ and $b_{L}$ are signal modulation dependent pseudorange and carrier phase satellite biases, respectively; $\boldsymbol{e}$ is the direction vector; $\boldsymbol{\delta} \boldsymbol{X}$ is the position correction vector; $m$ is the mapping function which maps residual troposphere zenith wet delay $T_{Z}$ into the slant delay toward the satellite; $\mu_{i}$ is a frequency-dependent ionosphere scaling factor; $I$ is the slant ionosphere delay; $\lambda$ is the wavelength of the corresponding carrier; $N$ is an integer carrier phase ambiguity.

The undifferenced and uncombined functional model allows for multi-frequency data processing, the inclusion of ionosphere corrections (Banville et al. 2014), and efficient phase ambiguity resolution at the user (Shi and Gao 2014). However, this research is limited to a dual-frequency case, because of the missing receiver antenna phase center offset (PCO) and variation (PCV) model for Galileo E5b and E6 frequencies. For E1 and E5a frequencies, we adopted PCO and PCV models for L1 and L5 GPS frequencies, respectively, due to the common frequency bands. Because of the limited quality of real-time ionospheric models (Nie et al. 2018), which significantly affects the positioning accuracy, we considered neither a single-frequency positioning nor did we impose ionosphere constraints. Finally, we did not perform a real-time carrier phase ambiguity resolution, which is still a challenging task even for GPS (Laurichesse and National 2011).

\section{Processing variants and strategy}

We used E1 and E5a Galileo observations collected from January 1 to 7, 2019 and stored in RINEX 3.03 files from 20 IGS stations distributed worldwide (Fig. 5). We further used IGS Real-Time Service (RTS, http://www.igs.org/ rts) as a source of real-time broadcast ephemeris (RTCM3EPH stream), real-time orbit and clock corrections, and observation specific code and phase biases (CLK93 stream). These products were recorded using BKG Ntrip Client v 2.12 (BNC) in ASCII files. The processing was done in the GNSS-WARP software (Hadas 2015) in the simulated real-time mode, which fully reflects true realtime conditions. The software allowed us to process the same dataset in ten different variants (Table 1). They are combinations of static and kinematic mode, pseudorangeonly or pseudorange with carrier phase processing, application of broadcast ephemeris (BRDC), RTS products or final MGEX products. Moreover, in order to compare Galileo-only with GPS-only positioning performance, we reproduce all ten processing variants using GPS observation on L1 and L2 frequencies.

In case of pseudorange-only positioning, the functional model is based on (1); however, the residual troposphere zenith wet delay term $T_{Z}$ was removed, so that $T_{Z}$ was not estimated. For $n$ satellites in view, it was possible to form $2 n$ observation equations in each epoch, while $4+n$ parameters were estimated: three coordinates, one receiver clock offset and $n$ slant ionospheric delays $I^{s}$. Therefore, at least 4 satellites were required to obtain a solution. In case of pseudorange and carrier phase positioning the $4 n$ equations were formed, but additional $2 n$ carrier phase ambiguities $N_{i}^{s}$ and one $T_{Z}$ had to be estimated, so at least 5 satellites in view were required.

In the static mode, coordinates and their estimated standard deviations (STD) were propagated (by means of epoch-wise least squares adjustment) from one epoch to another. In the kinematic mode, STDs of coordinates were reset every epoch to $100 \mathrm{~m}$. We calculated broadcast orbits and clocks using the Galileo Integrity Navigation Message (I/NAV) because real-time corrections are referred to the I/NAV ephemeris (RTCM 2011). Real-time precise orbit and clock corrections were applied following the algorithm described in Hadas and Bosy (2015), taking into account the matching of Issue of Data (IOD) parameter (Kazmierski et al. 2018). For the post-processing mode, we used MGEX orbits and clock products provided by the CODE analysis center. In the latter case, we also applied the satellite PCO and PCV corrections, because final products are referred to the satellite center of mass. For other cases, satellite PCO and PCV were not applied because the broadcast orbits and real-time CNES products are referred to the satellite antenna phase center (Montenbruck et al. 2015; Kazmierski et al. 2018). Code and carrier phase satellite biases were applied only together with precise orbit and clock corrections, i.e., those from RTS or MGEX. We assumed that variants based on broadcast ephemeris were "offline", i.e., no external corrections were provided. More details on the processing strategy can be found in Table 2 . 


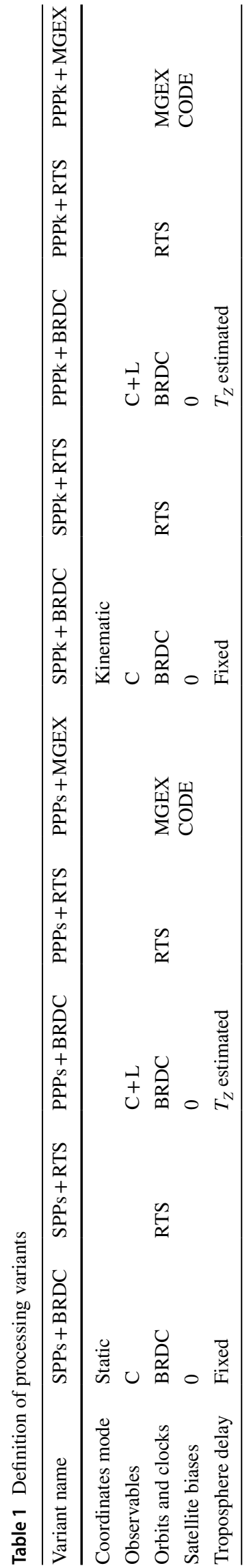

Galileo-only static positioning

The comparison of a coordinate time series obtained in the static mode with all processing variants is presented in Fig. 6. As expected, PPPs solutions perform significantly better than SPPs solutions, especially for the Up component and when taking into account the convergence time. Nevertheless, in all variants, except for SPPs + BRDC, the horizontal coordinates converge below the level of $0.1 \mathrm{~m}$ after half of a day. Moreover, remarkably accurate results are obtained in PPPs + BRDC, with the convergence time comparable to the PPPs + RTS solution, but still longer than in the PPPs + MGEX solution.

The accuracy of all daily static coordinates obtained with Galileo-only pseudorange positioning is illustrated in Fig. 7. Overall, the repeatability of SSPs + RTS solutions is better than of SPPs + BRDC solutions, especially for the East component. Although we notice station-specific offsets, the average bias is close to zero for both solutions in North and East components. For the Up component, both solutions are biased by about $0.25 \mathrm{~m}$, which can be attributed to the mismodeling of the tropospheric delay. In general, the accuracy better than $0.3 \mathrm{~m}$ and $1.0 \mathrm{~m}$ is obtained from SPPs + BRDC for the horizontal and vertical components, respectively. Application of RTS products improves the accuracy by $37 \%$ and $16 \%$, respectively.

Figure 8 shows the accuracy of all daily static positions obtained using Galileo-only pseudorange and carrier phase positioning. The use of MGEX and RTS products result in the most accurate coordinates at the level of a few centimeters for horizontal components and the sub-decimeter level for the vertical component. The PPPs + BRDC solution is not that accurate, but still, a level of $0.1 \mathrm{~m}$ and $0.2 \mathrm{~m}$ is usually achieved for the horizontal and vertical components, respectively. We notice significantly worse performance for station MKEA, because of several long periods with an insufficient number of tracked satellites during the time of the experiment.

\section{Galileo-only kinematic positioning}

The comparison of the coordinate time series obtained in the kinematic mode with all processing variants is illustrated in Fig. 9. Pseudorange-only solutions are characterized by a significant degradation of accuracy compared to PPPk solutions, while the latter ones are very consistent with each other. This is also reflected by the distribution of coordinate STDs, as presented in Fig. 10. Although SPPk variants are obtained for over $80 \%$ of solutions, less than $50 \%$ of them have STDs below $0.5 \mathrm{~m}$ and $1.0 \mathrm{~m}$ for 
Table 2 Summary of the processing strategy

\begin{tabular}{ll}
\hline A priori sigma of coordinates & $100 \mathrm{~m}$ \\
A priori sigma of observations & $0.3 \mathrm{~m}$ for pseudorange, $0.01 \mathrm{~m}$ for carrier phase \\
Frequencies & GPS L1, L2 or Galileo E1, E5a \\
Observables weighting & Elevation $(\varepsilon)$ dependent weighting: $\sin (\varepsilon)$ \\
Elevation cut-off angle & $5^{\circ}$ \\
Sampling rate & $30 \mathrm{~s}$ \\
Receiver clock offset & estimated as white noise, a priori sigma $=10^{5}[\mathrm{~m}]$ \\
Troposphere delay modeling & A priori ZTD from UNB3 m model (Leandro et al. 2008); Niell mapping function (Niell 1996); \\
& $T_{Z}$ estimated as random walk (4 mm/ $\sqrt{\text { hour })}$ \\
Solution type & Daily, with float ambiguities \\
Correction models & Phase wind-up, relativistic delays, solid earth tides, ocean tide loadings (Gérard and Luzum 2010) \\
Receiver PCO and PCV & From igs14.atx; E1, E5a from GPS L1, L5, respectively \\
\hline
\end{tabular}
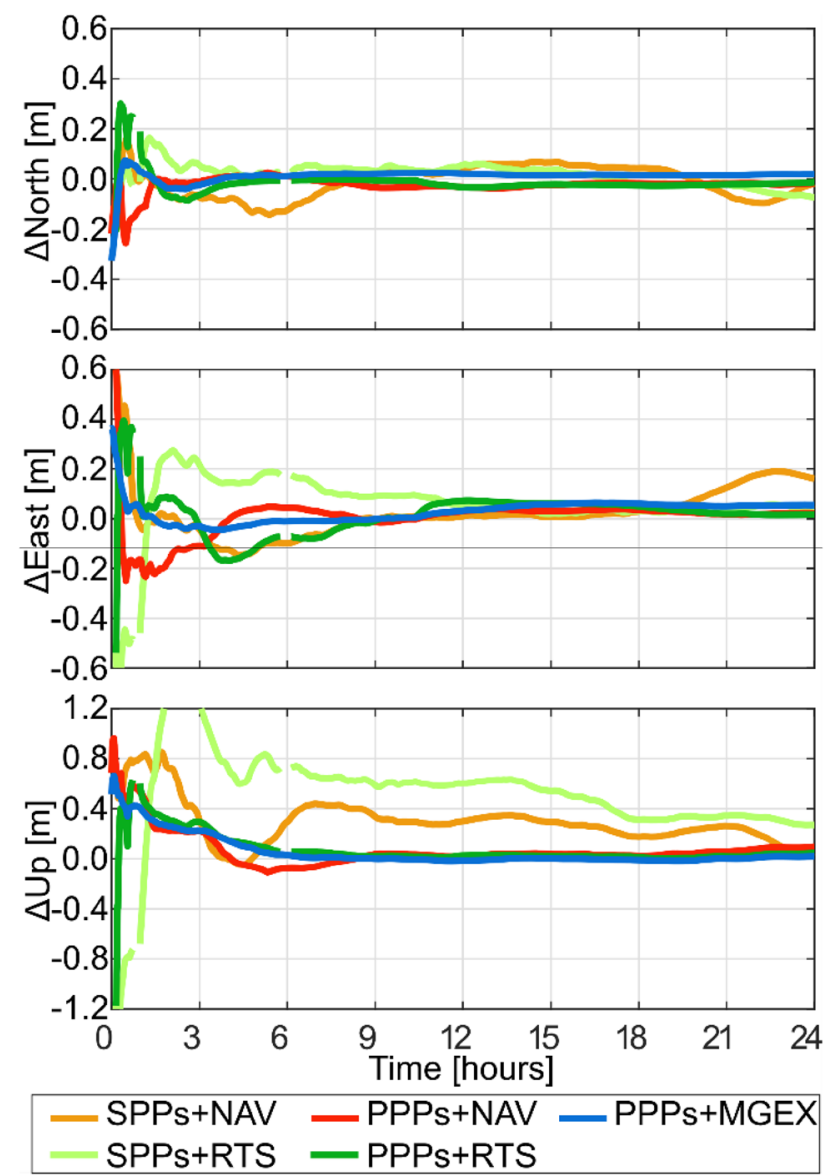

Fig. 6 Time series of coordinates obtained with Galileo-only static solution. Station WROC, January 1, 2019

the horizontal and vertical components, respectively. The availability of PPPk solutions varies from 59 to $67 \%$. In the cases of PPPk + BRDC and PPPk + RTS, more than half of the obtained solutions are characterized by a STD of horizontal components below $0.1 \mathrm{~m}$, while the STD of the vertical component is twice as large. Please note that
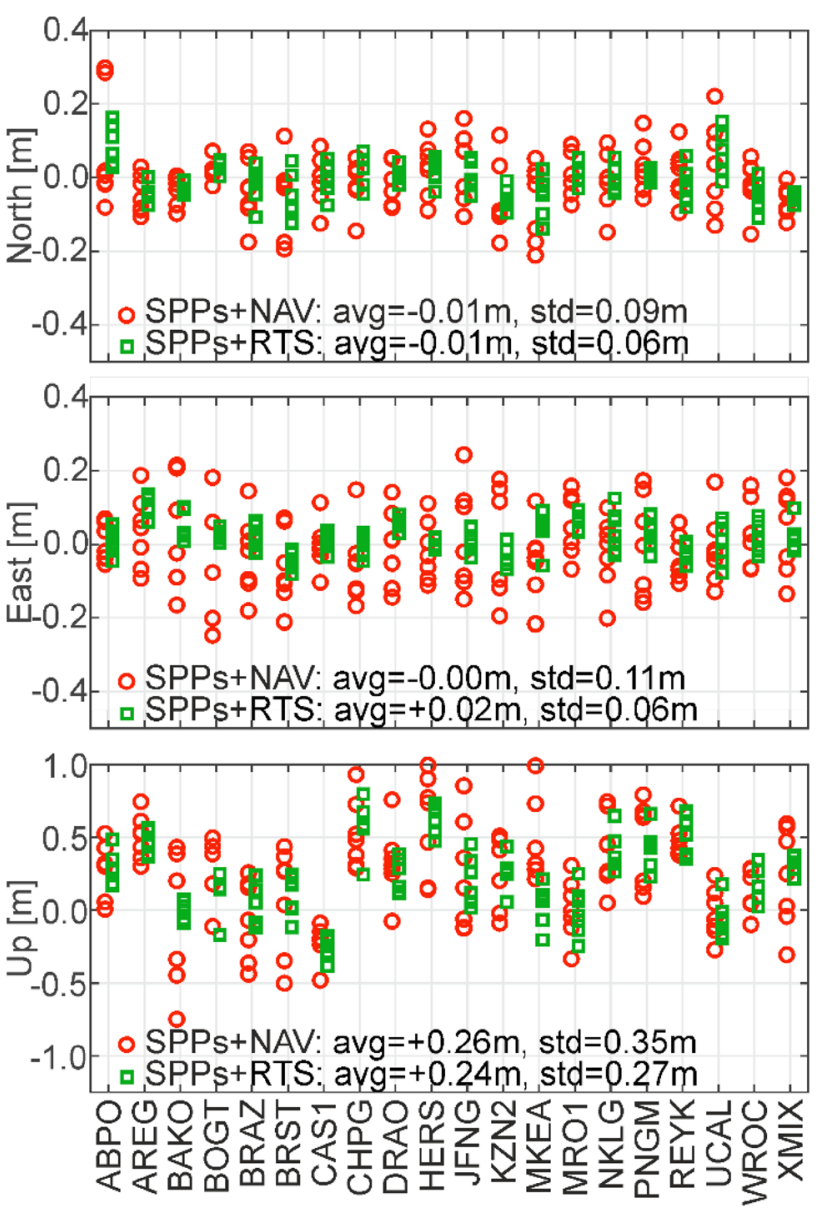

Fig. 7 Differences between coordinates obtained from Galileo-only daily static solutions and IGS weekly combined solution. January $1-7,2019$

these results include also initialization periods, i.e., outlier screening was not applied.

The advantage of using RTS is observed for the vertical component. However, in general, there are fewer PPPk + RTS solutions than PPPk + BRDC solutions, due 

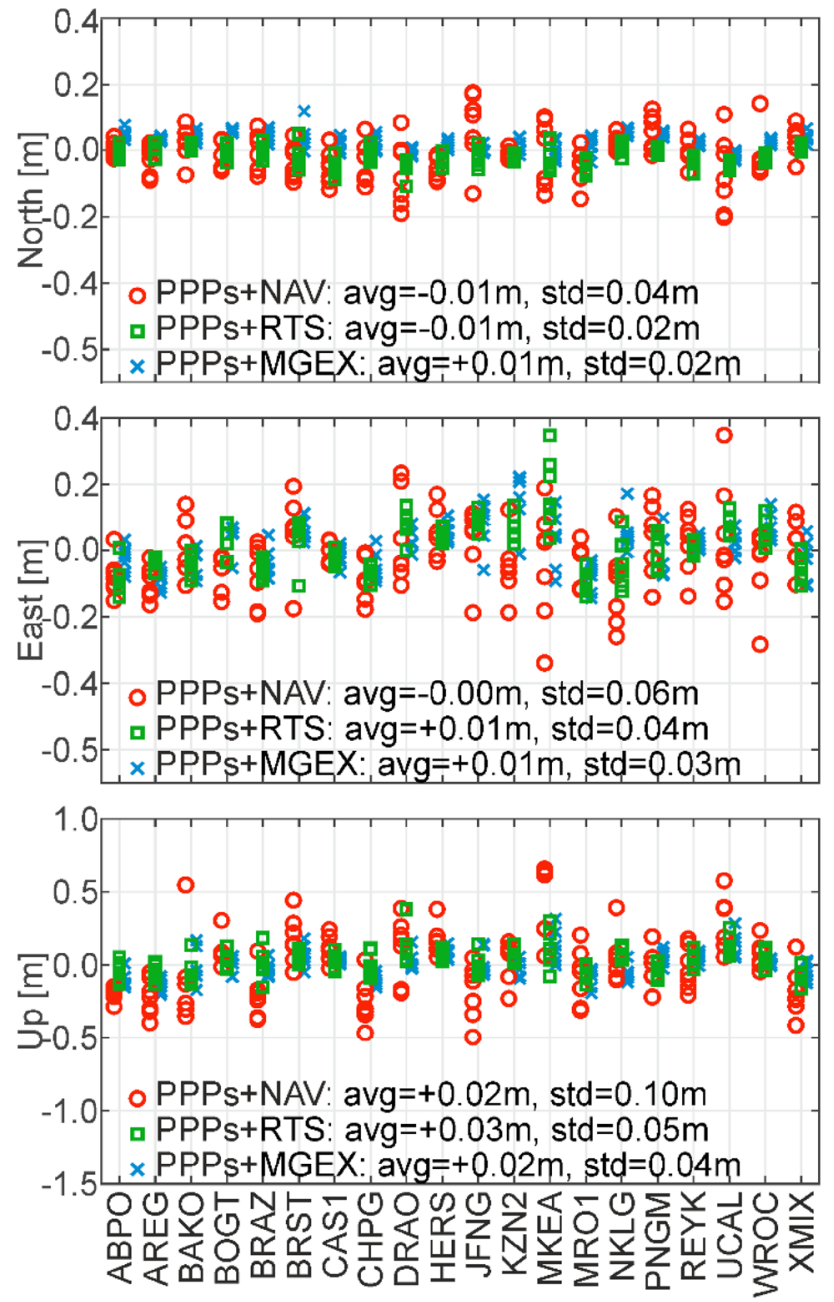

Fig. 8 Differences between coordinates obtained from Galileo-only pseudorange and carrier phase daily static solutions and IGS weekly combined solution. January 1-7, 2019

to the missing real-time corrections for the newest four Galileo satellites. The best performance is noticed for the PPPk + MGEX case. The availability of solutions is the same as in the PPPk + BRDC case, but the distribution of STDs is better than for the other two PPPk solutions.

The accuracy of kinematic coordinates from SPPk variants is shown in Fig. 11. In order to remove results from the initialization period, solutions with STDs of horizontal coordinates exceeding $1.0 \mathrm{~m}$ were removed from this analysis. North and East coordinate differences are within the range of $10 \mathrm{~m}$, with the standard deviation below $0.7 \mathrm{~m}$. For the vertical component, the accuracy is lower by the factor of 2. Similarly to the static mode, we observe station-specific offsets, related to the mismodeling of the tropospheric delay. The benefit of using RTS corrections is very limited.

The accuracy of PPPk positioning is shown in Fig. 12. A threshold equal to $0.2 \mathrm{~m}$ of the STD of horizontal coordinates was applied in order to remove solutions during
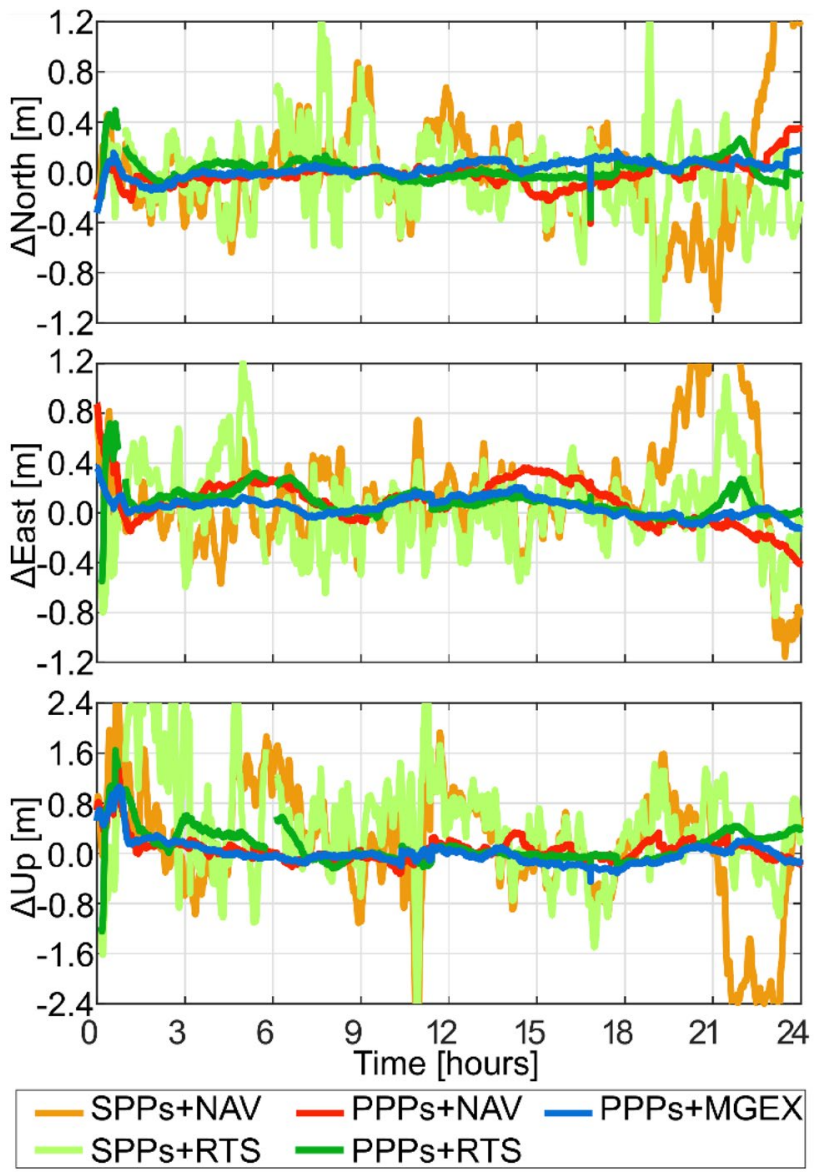

Fig. 9 Time series of coordinates obtained with Galileo-only kinematic solutions. Station WROC, January 1, 2019

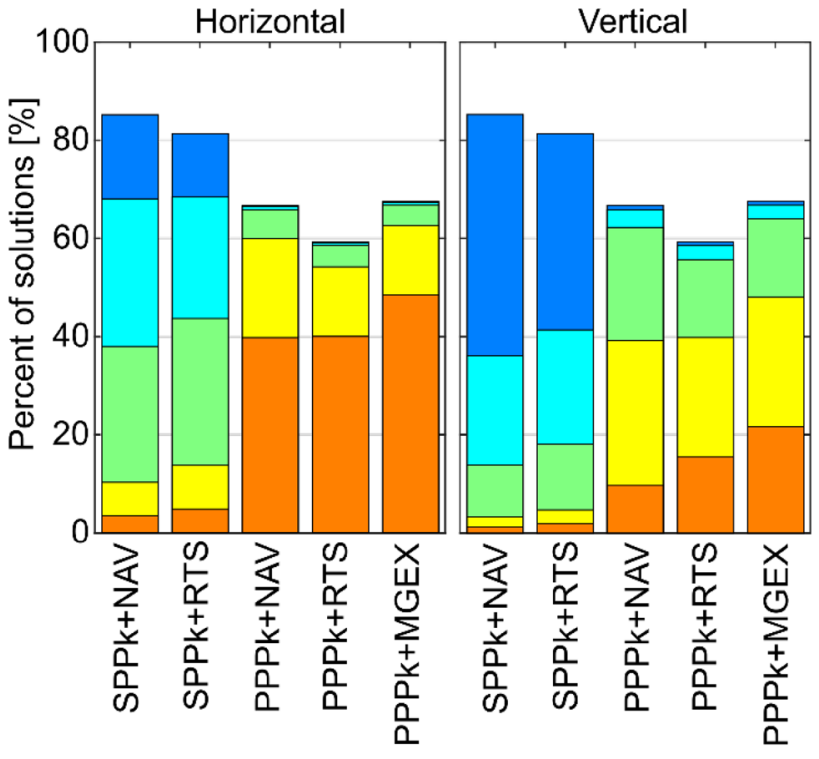

All $\square \sigma \leq 1.0 \mathrm{~m} \square \sigma \leq 0.5 \mathrm{~m} \square \sigma \leq 0.2 \mathrm{~m} \square \sigma \leq 0.1 \mathrm{~m}$

Fig. 10 Percent of Galileo-only kinematic solutions with the coordinate STD below a defined threshold 

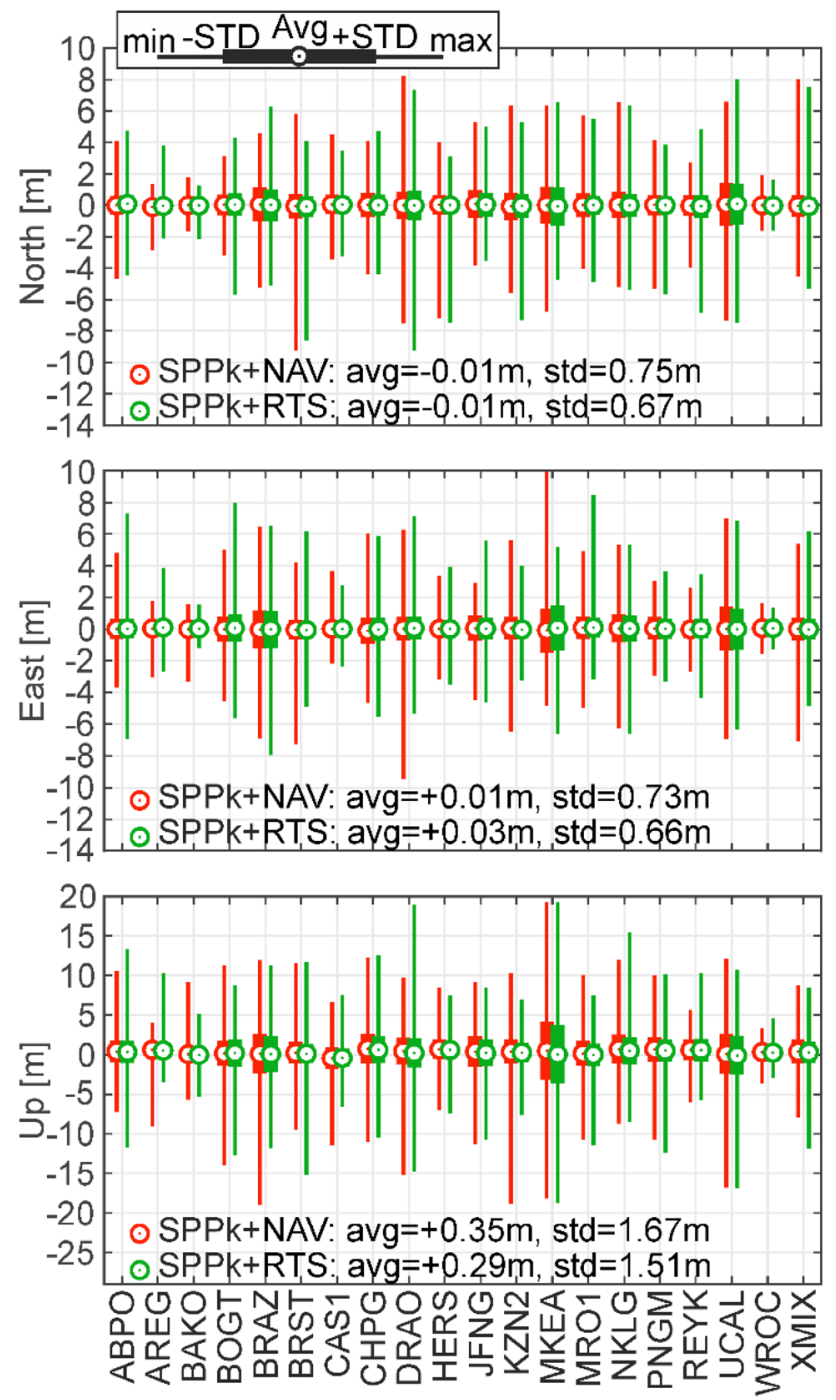

Fig. 11 Differences between coordinates obtained from Galileo-only pseudorange kinematic solutions and IGS weekly combined solution. January 1-7, 2019

the initialization period. The performance of PPPk + RTS and PPPk + MGEX is very similar. A bias close to zero is noticed for all components and the standard deviation of coordinate differences is $0.09 \mathrm{~m}, 0.14 \mathrm{~m}$ and $0.20 \mathrm{~m}$ for the North, East, and Up component, respectively. The accuracy of PPPk + BRDC solution is lower by more than $50 \%$ when compared to the other two PPPk solutions. Nevertheless, a submeter horizontal accuracy is achieved for PPPk + BRDC and the accuracy of the vertical component is better than $2.0 \mathrm{~m}$.
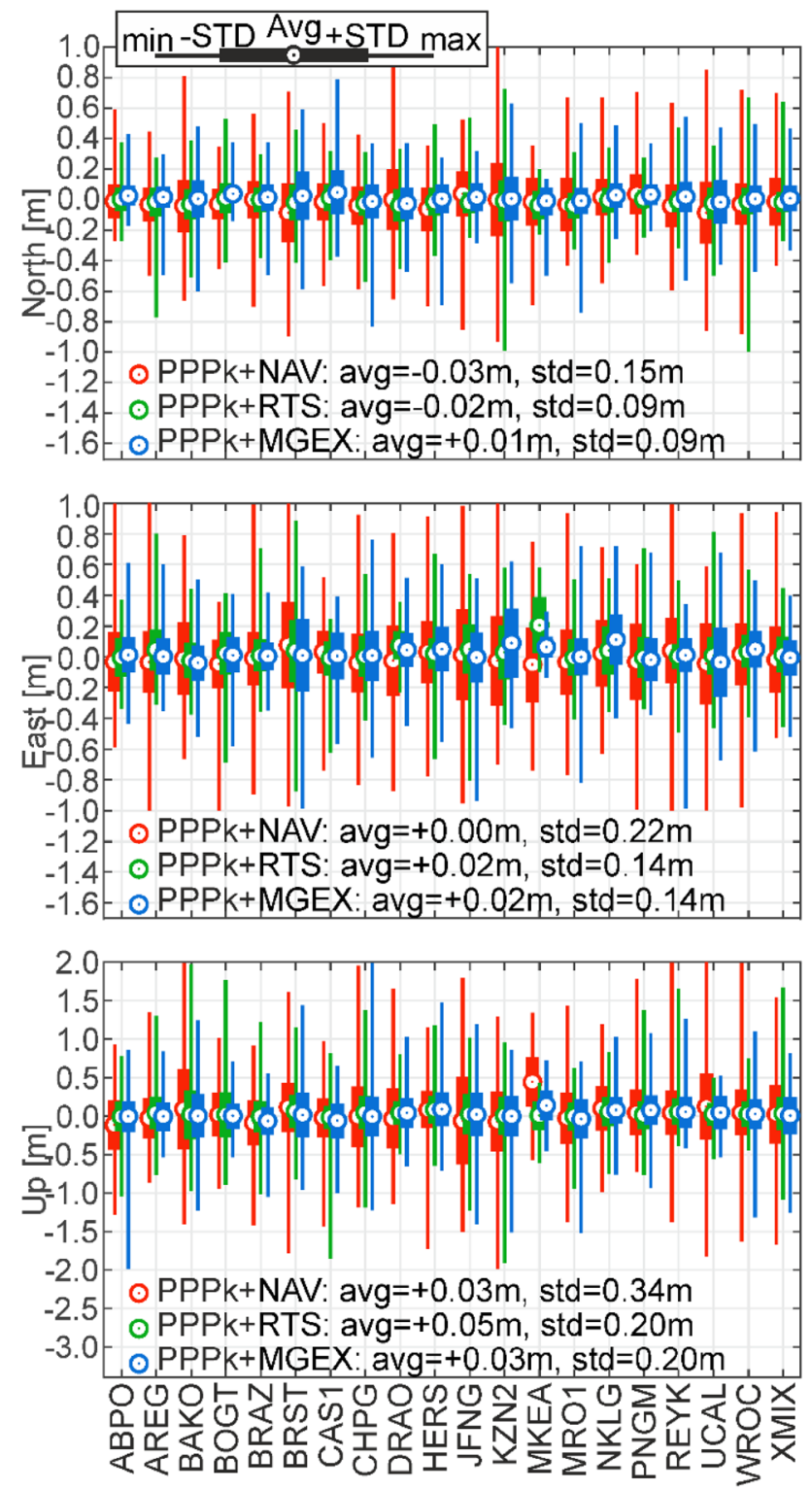

Fig. 12 Differences between coordinates obtained from Galileo-only pseudorange and carrier phase kinematic solutions and IGS weekly combined solution. January 1-7, 2019

\section{Galileo-only versus GPS-only positioning}

The overall comparison of GPS-only positioning accuracy with Galileo-only positioning accuracy, which was obtained using a dual-frequency daily static solution, is presented in Table 3. GPS-only PPP is still more accurate than Galileoonly PPP, both using RTS corrections and final products, which is due to the higher quality of satellite and clock products for GPS than for Galileo. On the other hand, a subdecimeter accuracy in both horizontal and vertical components is achieved with Galileo-only PPPs + BRDC, which is superior to GPS-only solution (Fig. 13, left panel). The 
Table 3 RMSE between estimated coordinates and IGS weekly combined solution for GPS-only and Galileo-only dual-frequency static positioning

\begin{tabular}{llllll}
\hline Processing variant & \multicolumn{2}{l}{ Horizontal $(\mathrm{m})$} & & \multicolumn{2}{c}{ Vertical $(\mathrm{m})$} \\
\cline { 2 - 3 } \cline { 6 - 6 } \cline { 6 - 7 } & GPS & Galileo & & GPS & Galileo \\
\hline SPPs + BRDC & 0.279 & 0.141 & & 0.701 & 0.433 \\
SPPs + RTS & 0.115 & 0.089 & & 0.418 & 0.365 \\
PPPs + BRDC & 0.197 & 0.067 & & 0.188 & 0.098 \\
PPPs + RTS & 0.031 & 0.049 & & 0.044 & 0.060 \\
PPPs + MGEX & 0.012 & 0.039 & & 0.013 & 0.040 \\
\hline
\end{tabular}
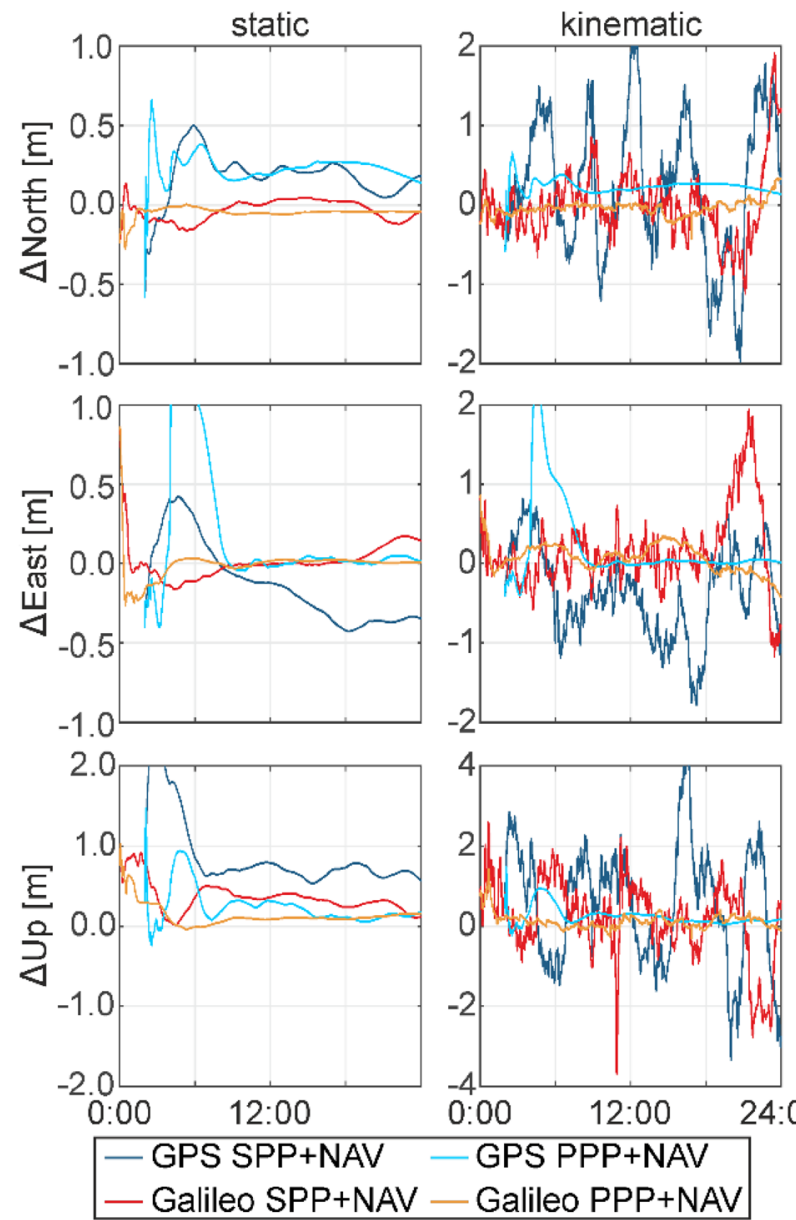

Fig. 13 Comparison of time series from GPS and Galileo solutions based on broadcast ephemeris. Station WROC, January 1, 2019

horizontal accuracy of Galileo-only solution is three times better than that of GPS-only solution, and the improvement in vertical component reaches $47 \%$. We attribute this to the high quality of Galileo broadcast orbits and clock offsets, which are ensured by frequent updates of Galileo ephemeris (Montenbruck et al. 2015). Moreover, due to the high precision of Galileo E1/E5 pseudoranges (Colomina et al. 2012),
Table 4 RMSE between estimated coordinates and IGS weekly combined solution for GPS-only and Galileo-only dual-frequency kinematic positioning

\begin{tabular}{llllll}
\hline Processing variant & \multicolumn{2}{l}{ Horizontal $(\mathrm{m})$} & & \multicolumn{2}{c}{ Vertical $(\mathrm{m})$} \\
\cline { 2 - 3 } \cline { 6 - 6 } & GPS & Galileo & & GPS & Galileo \\
\hline SPPk+BRDC & 1.269 & 1.045 & & 2.211 & 1.702 \\
SPPk + RTS & 0.921 & 0.938 & & 1.557 & 1.536 \\
PPPk + BRDC & 0.313 & 0.267 & & 0.352 & 0.337 \\
PPPk+RTS & 0.080 & 0.164 & & 0.103 & 0.210 \\
PPPk+MGEX & 0.158 & 0.153 & & 0.177 & 0.197 \\
\hline
\end{tabular}

Galileo-only SPPs + BRDC is more accurate than GPS-only solution (Fig. 13, left panel). The improvement reaches $49 \%$ and $38 \%$ for the horizontal and vertical component, respectively. When the RTS products are included in the SPP, the improvement is limited to $23 \%$ and $13 \%$, respectively. This is because RTS products for Galileo are still less accurate than GPS products (Kazmierski et al. 2018).

In the kinematic mode (Table 4), the performance of PPPk + MGEX solutions is comparable for GPS and Galileo. SPPk + BRDC for Galileo is more accurate by about $20 \%$ than for GPS (Fig. 13, right panel). GPS benefits from the RTS service; therefore, SPPk + RTS is more accurate by $27 \%$ than SPPk + BRDC and GPS PPPk + RTS is twice more accurate than the corresponding Galileo solution. In the PPPk + BRDC mode, the improvement of $15 \%$ and $4 \%$ is obtained with Galileo when compared to GPS, for the horizontal and vertical components, respectively (Fig. 13, right panel). For Galileo, the performance of PPPk + RTS and PPPk + MGEX is very similar. Surprisingly, for GPS the horizontal and vertical accuracy of PPPk + RTS is significantly better than that for PPPk + MGEX. This effect has been confirmed with an independent solution using RTKLib 2.4.2 software (Takasu and Yasuda 2009). We suppose this is caused by the long interval of MGEX clocks; therefore, a kinematic solution is contaminated by the linear interpolation of the satellite clock product.

\section{Conclusions}

Due to the rapid development of the Galileo space segment over last years and the accompanying support of the IGS, Galileo-only absolute positioning became available worldwide. The number of visible Galileo satellites is usually smaller by 1 to 3 compared to GPS. Although there are still two empty slots in the constellation, the global average PDOP is almost as good as that of GPS. In this study, a comprehensive evaluation of dual-frequency absolute positioning using the fully serviceable Galileo constellation was presented for the first time and compared to 
the performance of GPS positioning. Pseudorange-only results, as well as pseudorange and carrier phase results in real-time and post-processing modes, were evaluated.

We have demonstrated that static positioning using Galileo pseudoranges leads to an accuracy of a few decimeters and sub-meters for the horizontal and vertical components, respectively. In the kinematic case, the accuracy is better than $10 \mathrm{~m}$ for horizontal coordinates and better than $20 \mathrm{~m}$ in the vertical component. The Galileo static solutions based on broadcast ephemeris were superior to GPS results obtained using a corresponding strategy. Moreover, we noticed that pseudorange solutions using GPS and Galileo benefited from RTS products. The improvement varies from $10 \%$ for horizontal and vertical components in Galileo kinematic positioning up to 59\% for the horizontal components obtained in static GPS positioning.

Precise absolute positioning using pseudorange and carrier phase Galileo observations combined with RTS or MGEX products are not yet as good as the corresponding GPS solutions. In the static mode, the RMSE between estimated and reference coordinates does not exceed $0.05 \mathrm{~m}$ and $0.06 \mathrm{~m}$ for horizontal and vertical component, respectively. In the kinematic mode, the respective accuracies are better than $0.17 \mathrm{~m}$ and $0.21 \mathrm{~m}$. Remarkable results were achieved for Galileo PPP solutions based on the broadcast ephemeris. In the static mode, the RMSE of $0.07 \mathrm{~m}$ was achieved for the horizontal components and the RMSE of $0.10 \mathrm{~m}$ for the vertical component. This is superior to the corresponding GPS solutions by the factor of 3 and 2, respectively. In the kinematic mode, the Galileo horizontal and vertical components are better by $15 \%$ and $4 \%$ compared to those based on GPS.

The reduced Galileo pseudorange observation noise, great performance of onboard atomic clocks, and the corresponding high accuracy of Galileo broadcast ephemeris already allow us to obtain navigation solutions superior to these from GPS. However, further improvement in the quality of final and real-time products for Galileo is required. It should be expected that Galileo precise autonomous positioning will outperform GPS positioning when Galileo products reach the accuracy of GPS products and the Galileo constellation is finally completed.

Acknowledgements This work has been supported by the Polish National Science Center (UMO-2018/29/B/ST10/00382) and the Project EPOS-European Plate Observing System (POIR.04.02.0014-A0003/16) co-financed by the European Union from the European Regional Development Fund, Operational Programme Smart Growth 2014. We acknowledge the Centre National d'Études Spatiales (CNES) for providing real-time streams, the Bundesamt für Kartographie und Geodäsie (BKG) for providing the open-source BNC software, International GNSS Service for providing a variety of Galileo products and station reference coordinates.
Open Access This article is distributed under the terms of the Creative Commons Attribution 4.0 International License (http://creativeco mmons.org/licenses/by/4.0/), which permits unrestricted use, distribution, and reproduction in any medium, provided you give appropriate credit to the original author(s) and the source, provide a link to the Creative Commons license, and indicate if changes were made.

\section{References}

Angrisano A, Gaglione S, Gioia C, Massaro M, Troisi S (2013) Benefit of the NeQuick Galileo version in GNSS singlepoint positioning. Int J Navig Obs 2013, 302947. https://doi. org/10.1155/2013/302947

Banville S, Collins P, Zhang W, Langley RB (2014) Global and regional ionospheric corrections for faster PPP convergence. Navigation 6(2):115-124. https://doi.org/10.1002/navi.57

Cai C, Gao Y, Pan L, Dai W (2014a) An analysis on combined GPS/ COMPASS data quality and its effect on single point positioning accuracy under different observing conditions. Adv Space Res 54(5):818-829. https://doi.org/10.1016/J.ASR.2013.02.019

Cai C, Luo X, Liu Z, Xiao Q (2014b) Galileo signal and positioning performance analysis based on four IOV satellites. J Navig 67(5):810-824. https://doi.org/10.1017/S037346331400023X

Cai C, Gao Y, Pan L, Zhu J (2015) Precise point positioning with quad-constellations: GPS, BeiDou, GLONASS and Galileo. Adv Space Res 56(1):133-143. https://doi.org/10.1016/J. ASR.2015.04.001

Chatre E, Verhoef P (2018) Galileo Programme status update. In: Proceedings of ION GNSS 2018, Institute of Navigation, Miami, FL, USA, September 24-28, pp 733-767

Colomina I et al (2012) Galileo's surveying potential E5 pseudorange precision. GPS World 23:18-33

Delva P, Puchades N, Schönemann E, Dilssner F, Courde C, Bertone S, Gonzalez F, Hees A, Le Poncin-Lafitte Ch, Meynadier F (2015) A gravitational redshift test using eccentric Galileo satellites. Phys Rev Lett 121(23):231101. https://doi.org/10.1103/ PhysRevLett.121.231101

Elsobeiey M, Al-Harbi S (2016) Performance of real-time precise point positioning using IGS real-time service. GPS Solut 20(3):565-571. https://doi.org/10.1007/s10291-015-0467-z

European Space Agency (2010) European GNSS (Galileo) open service signal in space interface control document. Eur Union. https://doi.org/10.2768/1968

Fu W, Huang G, Zhang Y et al (2019) Multi-GNSS combined precise point positioning using additional observations with opposite weight for real-time quality control. Remote Sens 11(3):311. https://doi.org/10.3390/rs11030311

Gérard P, Luzum B (2010) IERS conventions (2010). (IERS Technical Note; 36) Frankfurt am Main: Verlag des Bundesamts für Kartographie und Geodäsie, 2010. p 179. ISBN 3-89888-989-6

Hadas T (2015) GNSS-warp software for real-time precise point positioning. Artif Satell 50(2):59-76. https://doi.org/10.1515/ arsa-2015-0005

Hadas T, Bosy J (2015) IGS RTS precise orbits and clocks verification and quality degradation over time. GPS Solut 19(1):93105. https://doi.org/10.1007/s10291-014-0369-5

Kaplan ED, Hegarty CJ (2017) Understanding GPS/GNSS. Principles and Applications. Artech House, London

Kazmierski K, Sośnica K, Hadas T (2018) Quality assessment of multi-GNSS orbits and clocks for real-time precise point positioning. GPS Solut 22:11. https://doi.org/10.1007/s1029 1-017-0678-6

Laurichesse D, National C (2011) The CNES real-time PPP with undifferenced integer ambiguity resolution demonstrator. In: 
Proceedings of ION GNSS 2011, Institute of Navigation, Portland, Oregon, USA, September 19-23, pp 654-662

Leandro RF, Langley RB, Santos MC (2008) UNB3m_pack: a neutral atmosphere delay package for radiometric space techniques. GPS Solut 12(1):65-70. https://doi.org/10.1007/s10291-007-0077-5

Liu G, Zhang X, Li P et al (2019) Improving the performance of Galileo uncombined precise point positioning ambiguity resolution using triple-frequency observations. Remote Sens 11(3):341. https://doi.org/10.3390/rs11030341

Loyer S, Perosanz F, Mercier F et al (2012) Zero-difference GPS ambiguity resolution at CNES-CLS IGS Analysis Center. J Geod 86(11):991-1003. https://doi.org/10.1007/s00190-012-0559-2

Malys S, Jensen P (1990) Geodetic point positioning with GPS carrier beat phase data from the CASA UNO experiment. Geophys Res Lett 17(5):651-654

Montenbruck O, Steigenberger P, Hauschild A (2015) Broadcast versus precise ephemerides: a multi-GNSS perspective. GPS Solut 19(2):321-333. https://doi.org/10.1007/s10291-014-0390-8

Montenbruck O, Steigenberger P, Prange L et al (2017) The MultiGNSS Experiment (MGEX) of the International GNSS Service (IGS) - achievements, prospects and challenges. Adv Space Res 59(7):1671-1697. https://doi.org/10.1016/j.asr.2017.01.011

Montenbruck O, Steigenberger P, Hauschild A (2018) Multi-GNSS signal-in-space range error assessment-methodology and results. Adv Space Res 61(12):3020-3038. https://doi.org/10.1016/j. asr.2018.03.041

Nie Z, Yang H, Zhou P et al (2018) Quality assessment of CNES real-time ionospheric products. GPS Solut 23:11. https://doi. org/10.1007/s10291-018-0802-2

Niell AE (1996) Global mapping functions for the atmosphere delay at radio wavelengths. J Geophys Res Solid Earth 101(B2):32273246. https://doi.org/10.1029/95JB03048

Odolinski R, Teunissen PJG, Odijk D (2014) First combined COMPASS/BeiDou-2 and GPS positioning results in Australia. Part I: single-receiver and relative code-only positioning. J Spat Sci 59(1):3-24

Pan L, Zhang X, Li X, Li X, LuC Liu J, Wang Q (2017) Satellite availability and point positioning accuracy evaluation on a global scale for integration of GPS, GLONASS, BeiDou and Galileo. Adv Space Res 63(9):2696-2710. https://doi.org/10.1016/J. ASR.2017.07.029

Parkinson BW, Axelrad P (1988) Autonomous GPS integrity monitoring using the pseudorange residual. Navigation 35(2):255-274. https://doi.org/10.1002/j.2161-4296.1988.tb00955.x

Paziewski J, Wielgosz P (2014) Assessment of GPS + Galileo and multi-frequency Galileo single-epoch precise positioning with network corrections. GPS Solut 18(4):571-579. https://doi. org/10.1007/s10291-013-0355-3

Píriz R, Martín-peiró B, Romay-merino M (2005) The Galileo constellation design: a systematic approach. In: Proceedings of ION ITM 2005, Institute of Navigation, Long Beach, CA, USA, September 13-16, pp 1296-1306

RTCM (2011) RTCM Standard 10403.1 differential GNSS services version 3. Virginia, USA

Satirapod C, Rizos C, Wang J (2001) GPS single point positioning with SA off: how accurate can we get? Surv Rev 36(282):255-262. https://doi.org/10.1179/sre.2001.36.282.255

Schönemann E (2014) Analysis of GNSS raw observations in PPP solutions. Dissertation, Technische Universität Darmstadt

Shi J, Gao Y (2014) A comparison of three PPP integer ambiguity resolution methods. GPS Solut 18(4):519-528. https://doi. org/10.1007/s10291-013-0348-2
Steigenberger P, Montenbruck O (2017) Galileo status: orbits, clocks, and positioning. GPS Solut 21(2):319-331. https://doi. org/10.1007/s10291-016-0566-5

Takasu T, Yasuda A (2009) Development of the low-cost RTK-GPS receiver with an open source program package RTKLIB. In: International symposium on GPS/GNSS

Zumberge JF, Heflin MB, Jefferson DC, Watking MM, Webb FH (1997) Precise point positioning for the efficient and robust analysis of GPS data from large networks. J Geophys Res Solid Earth 102(B3):5005-5017. https://doi.org/10.1029/96JB03860

Publisher's Note Springer Nature remains neutral with regard to jurisdictional claims in published maps and institutional affiliations.

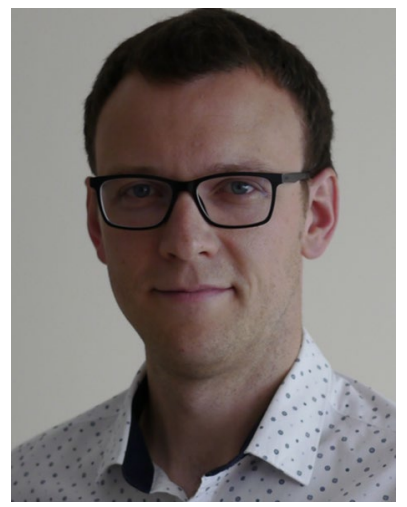

Tomasz Hadas received his Ph.D. in satellite geodesy in 2015 . He is working on the development of GNSS real-time precise positioning algorithms, troposphere modeling in real-time and application of atmosphere models into GNSS positioning.

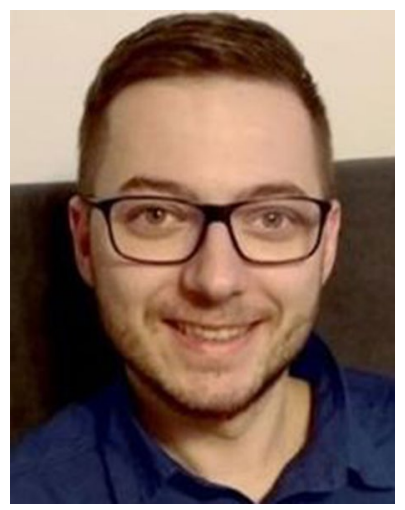

Kamil Kazmierski received his $\mathrm{Ph} . \mathrm{D}$. in satellite geodesy in 2018. His main field of interest is connected with the development of multi-GNSS real-time precise positioning algorithms and monitoring of the quality of real-time orbit and clock corrections.

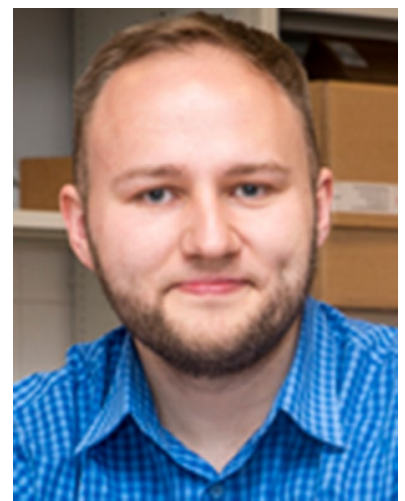

Krzysztof Sośnica graduated from the University of Bern, Switzerland in 2014 obtaining the Ph.D. degree of Science in Physics. His activities include precise orbit determination of GNSS and geodetic satellites, Earth's gravity field recovery, and the enhancement of the consistency between GNSS and SLR solutions. 